\title{
Is maximum primary tumor diameter still a prognostic factor in patients with nasopharyngeal carcinoma treated using intensity-modulated radiotherapy?
}

Yong Chen ${ }^{1 \dagger}$, Xue-Feng Hư ${ }^{2 \dagger}$, Yan Wang ${ }^{1}$, Hai-Yang Chen ${ }^{3}$, Lin Yang ${ }^{2}$, Li-Zhi Liư ${ }^{4}$, Chun-Yan Cui ${ }^{4}$, Dong-Sheng Liu ${ }^{5}$ and Shao-Bo Liang ${ }^{2^{*}}$

\begin{abstract}
Background: Intensity-modulated radiation therapy (IMRT) has represented a technical milestone that has facilitated the clinical implementation. The purpose of this study was to evaluate the prognostic value of maximum primary tumor diameter (MPTD) in patients with nasopharyngeal carcinoma (NPC) treated using IMRT.

Methods: Five-hundred and sixty-six patients with non-metastatic, histologically-confirmed NPC were retrospectively reviewed. MPTD was measured using magnetic resonance imaging (MRI). All patients were treated using IMRT; 87.5\% (456/521) of patients with Stage T3-T4/N1-N3 disease also received cisplatin-based chemotherapy. Receiver operating characteristic (ROC) curves were used to identify the optimal MPTD cut-off point and examine the prognostic value of combining MPTD with the current T classification criteria.

Results: Median follow-up for all patients was 36 months (range, 1-52 months). The 3-year overall survival (OS), failure-free survival (FFS), distant metastasis-free survival (DMFS), and local relapse-free survival (LRFS) rates for patients with a MPTD $\leq 41$ vs. $>41 \mathrm{~mm}$ were $96.1 \%$ vs. $85.4 \%, 93.7 \%$ vs. $74.7 \%, 96.1 \%$ vs. $79.7 \%$, and $98.1 \%$ vs. 92.9\%, respectively (all $P<0.05$ ). In multivariate analysis, MPTD was an independent prognostic factor for OS, FFS, DMFS and LRFS in all patients (all $P<0.05$ ). Among stage T3-T4 patients, the 3-year OS, FFS, DMFS, and LRFS rates for patients with a MPTD $\leq 41$ vs. $>41 \mathrm{~mm}$ were $96.9 \%$ vs. $84.5 \%, 95.4 \%$ vs. $73.5 \%, 96.1 \%$ vs. $79.2 \%$, and $99.3 \%$ vs. $92.6 \%$, respectively (all $P<0.05$ ). In multivariate analysis, MPTD was also an independent prognostic factor for OS, FFS and DMFS in stage T3-T4 patients (all $P<0.05$ ), and the difference in LRFS was almost statistically significant $(P=0.05)$. ROC curves verified that inclusion of MPTD improved the predictive value of the current $T$ classification criteria $(P<0.001)$.
\end{abstract}

Conclusions: MPTD was an independent prognostic factor in patients with NPC treated using IMRT, and significantly improved the prognostic value of the current T classification criteria for NPC.

Keywords: Nasopharyngeal carcinoma, Maximum primary tumor diameter, Intensity-modulated radiotherapy, Prognostic factor, TNM stage

\footnotetext{
* Correspondence: drshaoboliang@hotmail.com

${ }^{\dagger}$ Equal contributors

${ }^{2}$ Radiotherapy Department of Head \& Neck Carcinoma, Cancer Center, First People's Hospital of Foshan Affiliated to Sun Yat-sen University, 81 Lingnan Street North, Foshan, People's Republic of China

Full list of author information is available at the end of the article
} 


\section{Background}

Compared to other head and neck carcinomas, nasopharyngeal carcinoma (NPC) has a number of unique characteristics. Firstly, in contrast to its very low incidence in most other regions of the world, there is a high incidence of NPC in China and other countries in Southeast Asia [1]. Secondly, keratinizing squamous cell carcinoma is the major histological type in non-endemic areas; however, more than $95 \%$ of cases in the endemic areas are non-keratinizing carcinoma [2,3]. Thirdly, unlike other head and neck cancers, NPC is closely associated with Epstein-Barr viral infection $[4,5]$. Fourthly, radiotherapy (RT) is the first choice and main treatment method for non-metastatic NPC.

The introduction of intensity-modulated radiation therapy (IMRT) in the late 20th century represented a milestone in RT techniques. IMRT enables the tumor to receive higher dose of radiation, provides a more conformal dose distribution than two-dimensional RT (2DRT) and significantly reduces the dose to surrounding normal anatomical structures. Lee et al. initially reported that the 4-year local progression free survival rate for NPC after IMRT was 97\% (in 67 patients, $70 \%$ of whom had stage III-IV disease) [6]. Subsequently, other studies have confirmed that IMRT leads to excellent local control in NPC $[7,8]$.

The seventh edition of the American Joint Committee on Cancer (AJCC) staging system and the Chinese 2008 staging system for NPC are widely used in clinical work $[9,10]$. The $\mathrm{T}$ classifications of both systems are based on tumor invasion of anatomical structures and cranial nerve paralysis, and do not include tumor size. Primary gross tumor volume (GTV-P) is an important prognostic factor in NPC [11,12]. However, assessment of GTV-P is so time-consuming that it violates the basic requirements for staging systems to be simple and practical.

Liang et al. provided the first demonstration that maximum primary tumor diameter (MPTD) was an important prognostic factor for 5-year overall survival (OS), failure-free survival (FFS), distant metastasis-free survival (DMFS) and local relapse-free survival (LRFS) in NPC [13]. However, the techniques employed in that study have been replaced with superior techniques. Firstly, the patients received 2-DRT or three-dimensional conformal RT (3-DCRT) instead of IMRT, which not only offers local control, but also reduces RT-related toxicities in patients with NPC $[14,15]$. Secondly, the addition of concurrent chemotherapy to radiotherapy has improved the survival outcome of patients with loco-regionally advanced NPC [16-18]; however, only $46.6 \%(131 / 281)$ of the patients with Stage III-IVB disease received concurrent chemoradiotherapy [13]. Furthermore, it remains unknown whether the addition of
MPTD could improve the prognostic value of the $\mathrm{T}$ classification system for NPC.

On the basis of this premise, we initiated a retrospective study of a large cohort of patients to evaluate the prognostic value of MPTD in patients with NPC treated by IMRT and to determine whether the prognostic value of the current $\mathrm{T}$ classification system could be improved when combined with assessment of MPTD.

\section{Methods}

\section{Study population}

The Institutional Review Board of the Sun Yat-sen University Cancer Center approved the retrospective study. Written consent was waived, while oral consent was obtained via telephone and documented by telephone recording.

If the participants were at the age of 18 or over it, the oral consent was obtained from the participants. Otherwise, it would be obtained from their parents or guardians. Between November 2009 and December 2012, 566 consecutive patients with newly-diagnosed, histologically-proven, non-metastatic NPC who were treated at Sun Yat-sen University Cancer Center were included in this retrospective study. The cohort included 415 males and 151 females (male:female ratio, 2.7:1) with a median age of 46 years (range, 14-80 years). Histologically, $99.8 \%$ (565/566) of patients had non-keratinizing NPC; $0.2 \%(1 / 566)$ had keratinizing NPC. All patients underwent a pretreatment evaluation that included a complete medical history, physical and neurological examinations, hematology and biochemistry profiles, MRI scan of the nasopharynx and neck, chest radiography and abdominal sonography. Medical and imaging records were retrospectively reviewed, and all patients were restaged according to the 7th edition of the AJCC. The TNM stage distribution for all patients was $25.8 \%$ for T1, $17.5 \%$ for T2, 39.4\% for T3, and $17.3 \%$ for T4; $16.1 \%$ for N0, $60.2 \%$ for N1, $20 \%$ for N2, and 3.7\% for N3; $5.8 \%$ for stage I, $27.9 \%$ for stage II, $45.9 \%$ stage III, and $20.3 \%$ stage IVA-B.

\section{Imaging protocol}

All patients underwent MRI using a 1.5-Tesla system (Signa CV/i; General Electric Healthcare, Chalfont St. Giles, United Kingdom). The area from the suprasellar cistern to the inferior margin of the sternal end of the clavicle was examined using a head-and-neck combined coil. T1-weighted fast spin-echo images in the axial, coronal and sagittal planes (repetition time, 500-600 ms; echo time, $10-20 \mathrm{~ms} ; 22 \mathrm{~cm}$ field of view; $256 \times 512$ frequency matrix), and T2-weighted fast spin-echo MRI in the axial plane (repetition time, 4,000-6,000 ms; echo time, $95-110 \mathrm{~ms} ; 22 \mathrm{~cm}$ field of view; $256 \times 512$ frequency matrix) were obtained before injection of 
contrast material. After intravenous injection of gadopentetate dimeglumine $(0.1 \mathrm{mmol} / \mathrm{kg}$ body weight Gd-DTPA; Magnevist; Bayer-Schering, Berlin, Germany), spin-echo T1-weighted axial and sagittal sequences and spin-echo T1-weighted fat-suppressed coronal sequences were performed sequentially, using similar parameters to before injection. The section thickness was $3-4 \mathrm{~mm}$ with a $1 \mathrm{~mm}$ interslice gap for the sagittal plane, and $5 \mathrm{~mm}$ with a $1 \mathrm{~mm}$ interslice gap for the coronal and axial planes.

\section{Image assessment}

Two radiologists qualified in diagnostic imaging in China with $\geq 10$ years clinical experience focusing on head and neck carcinoma evaluated the MR images separately. Every two weeks, any disagreements were resolved by consensus. Tumors and soft tissue had intermediate signal intensity on pre-Gd-DTPA-T1 and T2-weighted images and enhanced intensity on postGd-DTPA T1-weighted images, with tumor replacing the normal anatomy of the structure. MPTD, defined as the maximum diameter of the continuous, uninterrupted tumor signal on post-Gd-DTPA T1-weighted images, was measured in the axial, coronal and sagittal planes; the largest value was recorded as the MPTD $[13,19]$.

\section{Treatment}

All patients were treated using IMRT; the protocol has previously been reported in detail $[20,21]$. The prescribed dose was 68-70 Gy to the planning target volume (PTV) of GTV-P, $60-66$ Gy to the PTV of the nodal gross tumor volume (GTV-N), 60-62 Gy to the PTV of CTV-1 (i.e. high-risk regions) and 54-56 Gy to the PTV of CTV-2 (i.e. low-risk regions and neck nodal regions) over 30-31 fractions. RT was delivered over one fraction daily, 5 days per week.

Based on institutional treatment guidelines, concurrent chemotherapy was recommended for Stage T1-2N1M0 disease and concurrent chemotherapy +/- induction chemotherapy or adjuvant chemotherapy for Stage III-IVb disease. Overall, platinum-based chemotherapy was administered to $87.5 \%(456 / 521)$ of patients with Stage T1-2N1M0 or Stage III-IVb disease. In the event of documented relapse or persistent disease, salvage treatments including after-loading, surgery or chemotherapy were provided when appropriate.

\section{Follow up and statistical analysis}

Patients were assessed every three months during the first two years, and every six months thereafter until censored (death, loss of follow-up or study termination). With regards to the measured indices, OS was measured from assignment to the date of death from any cause;
FFS indicated the first failure at any site; and LRFS and DMFS were recorded as the first local or remote failure, respectively. Distant metastases were diagnosed based on clinical symptoms, physical examinations and imaging methods including X-ray, bone scan, MRI, CT and abdominal sonography. Locoregional recurrence was established by fiberoptic endoscopy, biopsy and MRI.

All analyses were performed using SPSS software version 13.0 (SPSS, Chicago, IL, USA). Actuarial rates were calculated by the Kaplan-Meier method and the differences were compared using the log-rank test. Multivariate analyses with the Cox proportional hazards model were used to test for significant independent prognostic factors using a backward elimination strategy. All patients were randomly allocated to a training set $(n=189)$ and a test set $(n=377)$. Receiver operating characteristic (ROC) curve analysis was used to evaluate different cut-off points for MPTD in the training set. Then, the test set and all patients were stratified according to the optimum cut-off point. The area under the ROC curve was used to assess the prognostic value of MPTD. The criterion for statistical significance was set at $\alpha=0.05$ and $P$-values were based on two-sided tests.

\section{Results}

\section{Distribution of MPTD by T stage and survival rates}

The distribution of MPTD by T stage is presented in Figure 1. The median MPTD was $28.7 \mathrm{~mm}$ (range, $14-50 \mathrm{~mm})$ in T1, $33.3 \mathrm{~mm}(17-65.3 \mathrm{~mm})$ in T2, $39.7 \mathrm{~mm}$ in T3 $(14.3-77.6 \mathrm{~mm})$, and $59.6 \mathrm{~mm}$ in T4 $(24-121.5 \mathrm{~mm})$. The MPTD values varied widely within the same $\mathrm{T}$ stage, and overlapped between different $\mathrm{T}$ stages.

The median follow-up for all patients was 36 months (range, 1 to 52 months). In total, 9\% (51/566) of patients developed distant metastases, 5.3\% (30/566) developed locoregional recurrence and $7.4 \%(42 / 566)$ died. The 3 -year OS, DFS, DMFS and LRFS rates were 92.4\%, $86.9 \%, 90.3 \%$ and $96.4 \%$, respectively.

\section{Identification and prognostic verification of the optimal MPTD cut-off point}

The optimal cut-off point for MPTD with respect to OS in the training set $(n=189)$ was $41 \mathrm{~mm}$ (sensitivity $81.8 \%$, specificity $66.9 \%$; AUC [area under the ROC] = $0.74, P=0.007)$. Therefore, we selected a uniform cutoff point of $41 \mathrm{~mm}(>41 \mathrm{vs}$. $\leq 41 \mathrm{~mm})$ to classify the test set and all patients into high and low MPTD groups for survival analysis.

In the test set $(n=377)$, the 3-year OS, FFS, and DMFS rates for patients with a MPTD $\leq 41$ vs. $>41 \mathrm{~mm}$ were $95.1 \%$ vs. $89.2 \%, 92.7 \%$ vs. $74.1 \%$, and $95.5 \%$ vs. $77.9 \%$, respectively (all $P<0.05$ ). The 3 -year LRFS rates 


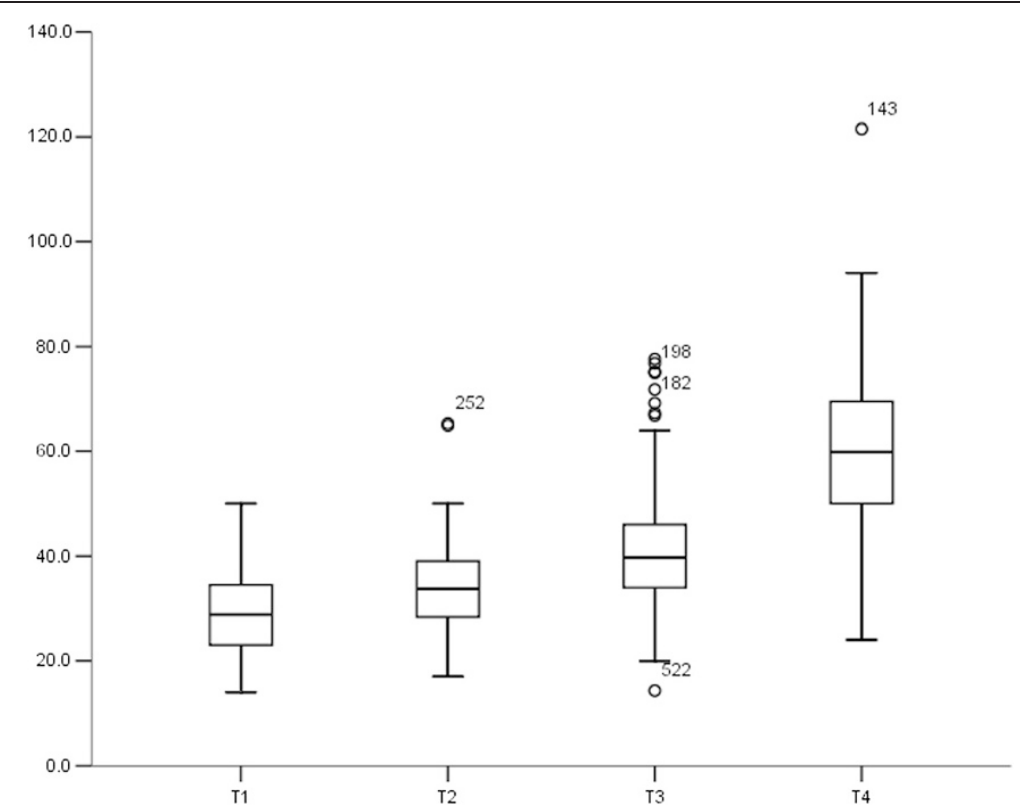

Figure 1 Distribution of maximum primary tumor diameter by T stage in 566 patients with NPC.

for patients with a MPTD $\leq 41$ vs. $>41 \mathrm{~mm}$ were $98.1 \%$ vs. $95.1 \%(P=0.191)$.

\section{Prognostic significance of MPTD in all patients}

In all patients $(n=566)$, the 3 -year OS, FFS, DMFS, and LRFS rates for patients with a MPTD $\leq 41$ vs. $>41 \mathrm{~mm}$ were $96.1 \%$ vs. $85.4 \%(P<0.001), 93.7 \%$ vs. $74.7 \% \quad(P<0.001), 96.1 \%$ vs. $79.7 \% \quad(P<0.001)$, and $98.1 \%$ vs. $92.9 \%(P=0.008)$, respectively (Figure 2$)$. The following parameters were included in the Cox proportional hazards model: age ( $\leq 45$ vs. $>45$ years), sex, chemotherapy (yes vs. no) and additional boosts (yes vs. no), T stage (T1-2 vs. T3-4), $\mathrm{N}$ stage (N0-1 vs. N2-3) and MPTD ( $\leq 41$ vs. $>41 \mathrm{~mm})$. MPTD was an independent prognostic factor for OS, FFS, DMFS and LRFS (all $P<0.05$; Table 1).

\section{Prognostic significance of MPTD in patients with advanced T classification}

The 321 patients with T3-T4 stage disease were divided into two subgroups: Group 1 (T3-T4 disease with a MPTD $\leq 41 \mathrm{~mm}$ ) and Group 2 (T3-T4 disease with a MPTD >41 mm). The 3-year OS, FFS, DMFS, and LRFS rates of the patients in Group 1 and Group 2 were $96.9 \%$ vs. $84.5 \%(P<0.001), 95.4 \%$ vs. $73.5 \%(P<0.001), 96.1 \%$ vs. $79.2 \%(P<0.001)$, and $99.3 \%$ vs. $92.6 \%(P=0.037$; Figure 3$)$. The following parameters were included in the Cox proportional hazards model: age ( $\leq 45$ vs. $>45$ years), sex, chemotherapy (yes vs. no), additional boosts (yes vs. no), $\mathrm{T}$ stage (T3 vs. $\mathrm{T} 4$ ), $\mathrm{N}$ stage ( $\mathrm{N0}-1$ vs. N2-3) and MPTD ( $\leq 41$ vs. $>41 \mathrm{~mm})$. MPTD was an independent prognostic factor for OS, FFS and DMFS (all
$P<0.05)$, and the difference between LRFS was almost statistically significant $(P=0.05$; Table 2$)$.

\section{Predictive value of T classification combined with MPTD vs. T classification alone}

ROC curves were used to compare the predictive value of T classification combined with MPTD vs. T classification alone. In all patients, the AUC for $\mathrm{T}$ classification combined with MPTD $(<41$ and $>41 \mathrm{~mm})$ was 0.70 , compared to 0.67 for $\mathrm{T}$ classification alone $(P<0.001$; Figure 4). These results indicate that $\mathrm{T}$ classification combined with MPTD is superior to $\mathrm{T}$ classification alone for predicting prognosis.

\section{Discussion}

IMRT has gradually replaced 2-DRT and 3-DCRT, and is currently the mainstream radiotherapy technique. As a result of the improved treatment outcomes provided in NPC by IMRT, it is necessary to reassess the prognostic factors identified by analyses of patients treated with 2DRT and 3-DCRT [3,22,23]. This study demonstrates that MPTD was an independent prognostic factor for OS, FFS, DMFS and LRFS in patients with NPC treated using IMRT. Combining MPTD with the current criteria significantly improved the prognostic value of the T classification system for NPC.

\section{Prognostic value of MPTD in patients with NPC treated by} IMRT

Larger MPTD values were more frequent in patients with higher $\mathrm{T}$ stage. However, the MPTD values varied widely within the same $\mathrm{T}$ stage, and overlapped between 


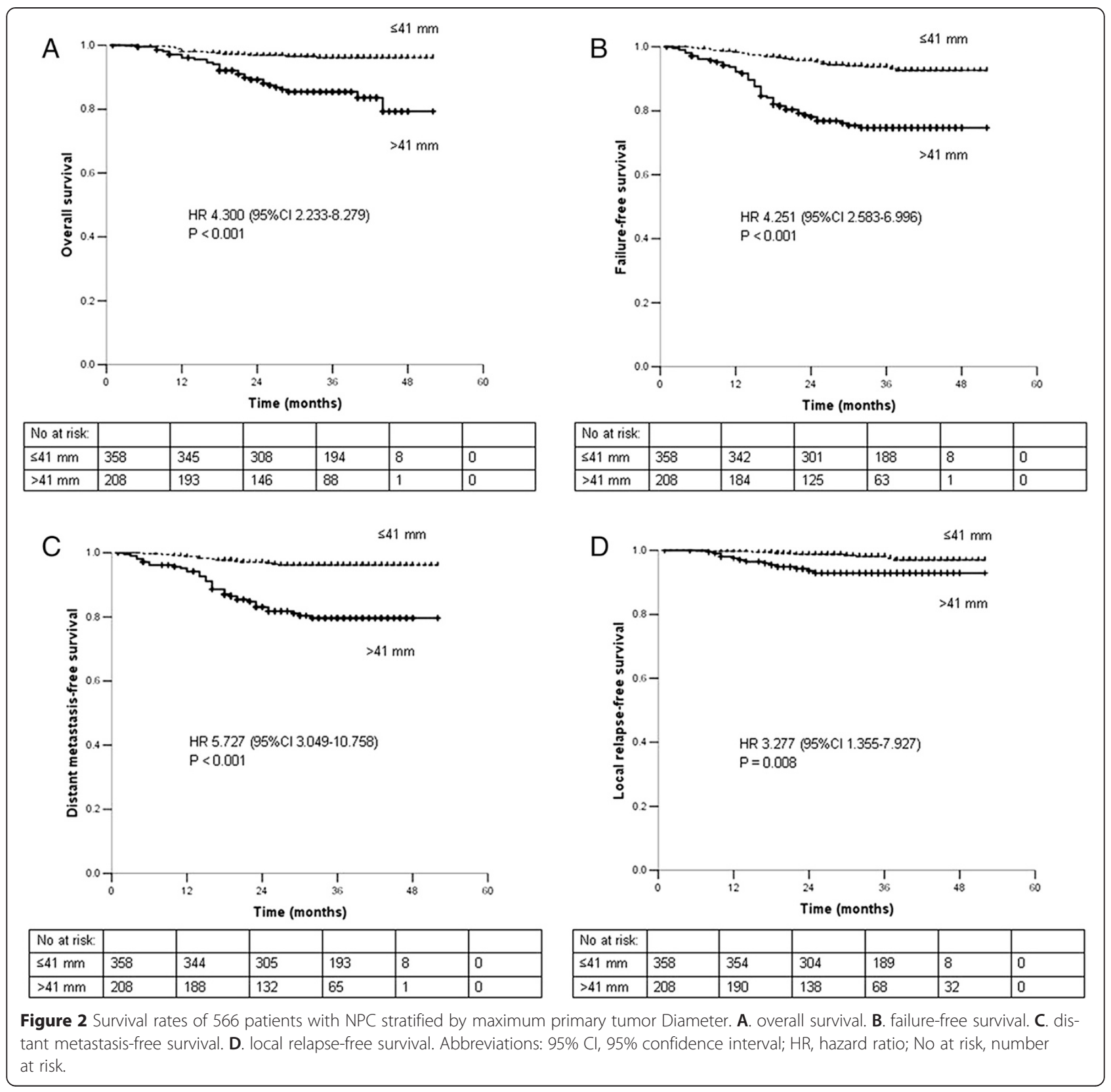

Table 1 Multivariate analyses of prognostic factors in $\mathbf{5 6 6}$ patients with nasopharyngeal carcinoma

\begin{tabular}{lllll}
\hline Endpoint & Variable & HR & $\mathbf{9 5 \%} \mathbf{C l}$ & $\boldsymbol{P}$-value \\
\hline Overall survival & MPTD & 4.300 & $2.233-8.279$ & $<0.001$ \\
Failure-free survival & MPTD & 3.968 & $2.402-6.559$ & $<0.001$ \\
& N stage & 1.802 & $1.111-2.923$ & 0.017 \\
Distant metastasis-free survival & MPTD & 5.153 & $2.729-9.729$ & $<0.001$ \\
& N stage & 2.329 & $1.334-4.064$ & 0.003 \\
Local relapse-free survival & MPTD & 3.277 & $1.355-7.927$ & 0.008 \\
\hline
\end{tabular}

Abbreviations: MPTD, maximum primary tumor diameter; $95 \% \mathrm{Cl}, 95 \%$ confidence interval; $\mathrm{HR}$, hazard ratio. different $\mathrm{T}$ stages. These findings are in agreement with a previous study [13], and indicate that the current $T$ classification does not accurately reflect tumor size in patients with NPC.

In the previous study by Liang et al., the patients were divided into three groups ( $\leq 30$ vs. $>30-50$ vs. $>50 \mathrm{~mm}$ ) on the basis of the balance of the distribution of the MPTD values [13]. However, the ROC curve analysis was used to define the optimal cut-off points in this study. A MPTD cut-off point of $41 \mathrm{~mm}$ was selected for predicting OS. This cut-off point was validated in the test set, in which the 3-year OS, FFS and DMFS rates 

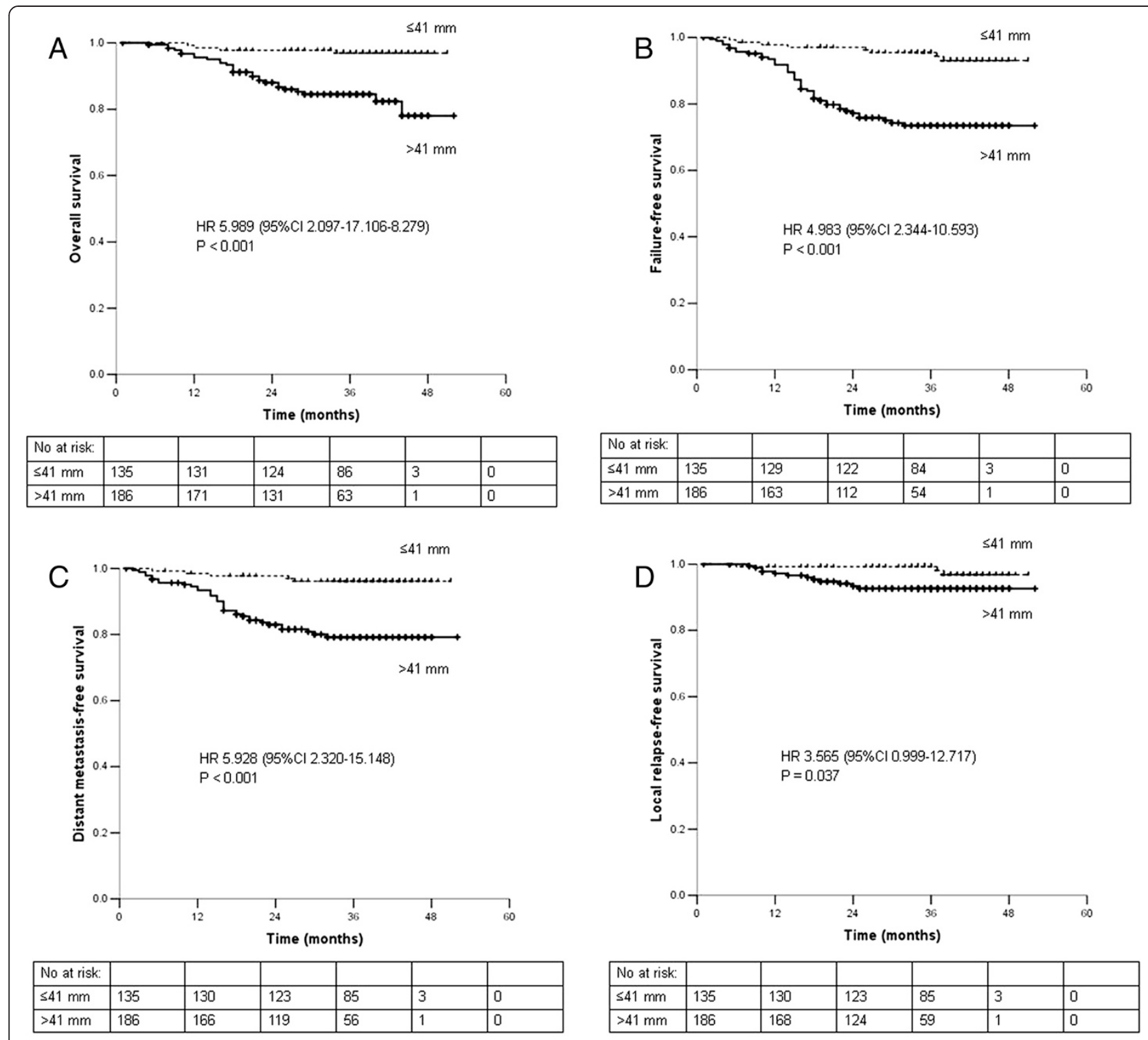

Figure 3 Survival rates of 321 patients with T3-T4 stage NPC stratified by maximum primary tumor diameter. A. overall survival. B. failure-free survival. C. distant metastasis-free survival. D. local relapse-free survival. Abbreviations: 95\% Cl, 95\% confidence interval; HR, hazard ratio; No at risk, number at risk.

Table 2 Multivariate analyses of prognostic factors in 321 patients with nasopharyngeal carcinoma and advanced $\mathbf{T}$ classification

\begin{tabular}{lllll}
\hline Endpoint & Variable & HR & $\mathbf{9 5 \%}$ Cl & $P$-value \\
\hline Overall survival & MPTD & 5.989 & $2.097-17.106$ & 0.001 \\
Failure-free survival & MPTD & 4.592 & $2.149-9.816$ & $<0.001$ \\
& N stage & 1.797 & $1.031-3.131$ & 0.039 \\
Distant metastasis-free survival & MPTD & 5.266 & $2.048-13.541$ & $<0.001$ \\
& N stage & 2.237 & $1.193-4.194$ & 0.012 \\
Local relapse-free survival & MPTD & 3.565 & $0.999-12.717$ & 0.050 \\
\hline
\end{tabular}

Abbreviations: MPTD, maximum primary tumor diameter; $95 \% \mathrm{Cl}, 95 \%$ confidence interval; $\mathrm{HR}$, hazard ratio. for patients with a MPTD $\leq 41$ were significantly better than those of patients with a MPTD $>41 \mathrm{~mm}$ (all $P<0.05)$.

MPTD (>41 vs. $\leq 41 \mathrm{~mm}$ ) was an independent prognostic factor for OS, FFS, DMFS and LRFS in both univariate and multivariate analysis in all patients treated using IMRT. Liang et al. previously reported that MPTD ( $\leq 30$ vs. $>30-50$ vs. $>50 \mathrm{~mm}$ ) was also an independent prognostic factor for OS, FFS, DMFS and LRFS in patients with NPC treated using 2-DRT or 3-DCRT [13]. Larger tumors may contain higher numbers of clonogenic tumor cells, possess larger areas of tumor hypoxia that promote resistance to radiotherapy and 


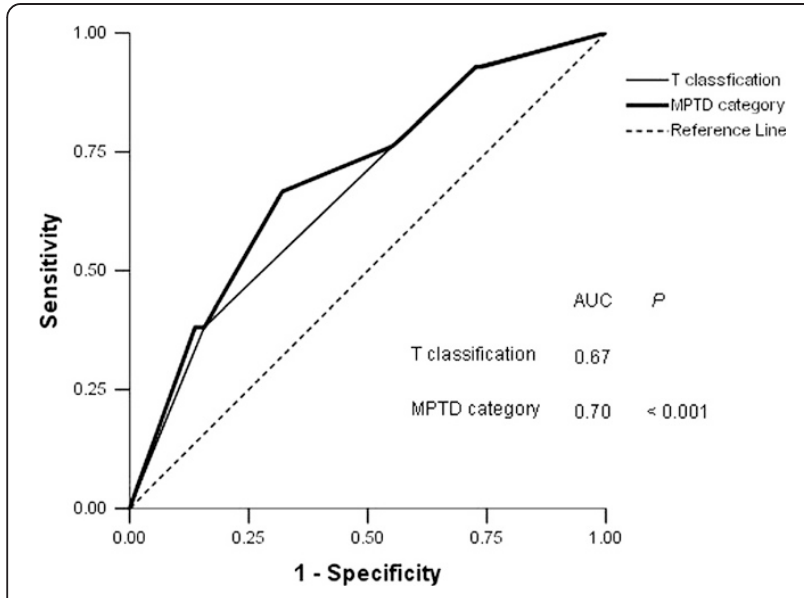

Figure 4 Receiver operator characteristic (ROC) curves for all patients with NPC ( $n=566)$ when stratified by T classification combined with maximum primary tumor diameter (MPTD) and by $T$ classification alone.

chemotherapy, or be associated with increased risk of distant micrometastases [24].

\section{Prognostic value of adding MPTD to the current T classification}

In the stratified analysis of patients with stage T3-T4 disease, MPTD was an independent prognostic factor for OS, FFS and DMFS (all $P<0.05$ ), and the difference in LRFS was nearly statistically significant $(P=0.05)$. Similar results were also observed in patients with NPC treated using 2-DRT or 3-DCRT [13]. This indicates that although advanced $\mathrm{T}$ classification disease is usually associated with poorer local control and shorter survival, patients within the same $\mathrm{T}$ classification with different MPTD values may have a different prognosis.

The current $T$ classification does not include an assessment of tumor size, which this study demonstrates is an important prognostic factor in patients with NPC. Compared to the previous study by Liang et al., in addition to employing ROC curves analysis, we also investigated whether the prognostic value of the current $T$ classification could be improved by adding MPTD. This study demonstrates that including MPTD in the current $\mathrm{T}$ classification enables superior prognostication compared to $\mathrm{T}$ classification alone $(P<0.001)$.

\section{Comparison of MPTD with GTV-P}

In 1997, Chua et al. reported that GTV-P values varied widely within each $\mathrm{T}$ stage and represented an independent prognostic factor for local control in NPC, which appeared to be more predictive than Ho's T stage classification [11]. Subsequently, a number of studies confirmed that GTV-P was an important prognostic factor in NPC $[12,25,26]$. Guo et al. reevaluated the prognostic value of GTV-P in 694 patients with NPC treated by IMRT, and confirmed that GTV-P was an independent prognostic factor that significantly improved the prognostic validity of $\mathrm{T}$ stage system [27].

MPTD and GTV-P, indexes reflecting the primary tumor size, are both important prognostic factors in patients with NPC. Compared to GTV-P, MPTD provides a less accurate assessment of tumor size; however, MPTD is quicker and easier to measure. Therefore, MPTD may be more convenient in clinical work and suitable for incorporation into the TNM staging system.

To the best of our knowledge, this is the first study to investigate the prognostic value of MPTD in patients with NPC treated by IMRT in a large number of patients. These results may help to refine the current $\mathrm{T}$ staging system for NPC. However, this was a retrospective study based on single-institution data, which needs to be confirmed by further multicentre studies.

\section{Conclusions}

This study is the first attempt to evaluate the prognostic value of MPTD in patients with NPC treated by IMRT. Our analyses demonstrate that MPTD is also an independent prognostic factor for OS, FFS, DMFS and LRFS in patients with NPC treated by IMRT. Addition of MPTD may help to refine the prognostic value of the current staging system for NPC and assist with treatment strategy selection.

\section{Competing interests}

The authors declare that they have no competing interests.

\section{Authors' contributions}

YC and XFH participated in literature research, study design, data collection, data analysis, interpretation of findings and the draft of the manuscript. YW, HYC and LY carried out the data collection. LZL and CYC reviewed MR images. DSL performed the statistical analysis. SBL contributed with study design, data collection, interpretation of findings and critical edit of the manuscript. All authors read and approved the final manuscript.

\section{Acknowledgements}

The study was supported by grants from the Science Foundation from the Sci-Tech Office of Guangdong Province (No. 2010B080701014) and the Science Foundation from the Health Bureau of Foshan City (No. 2013064).

\section{Author details}

'Department of Radiation Oncology, Sun Yat-sen University Cancer Center; State Key Laboratory of Oncology in South China; Collaborative Innovation Center for Cancer Medicine, Guangzhou, People's Republic of China.

${ }^{2}$ Radiotherapy Department of Head \& Neck Carcinoma, Cancer Center, First People's Hospital of Foshan Affiliated to Sun Yat-sen University, 81 Lingnan Street North, Foshan, People's Republic of China. ${ }^{3}$ Department of Radiation Oncology, the Sixth Affiliated Hospital of Sun Yat-sen University, Guangzhou, People's Republic of China. ${ }^{4}$ State Key Laboratory of Oncology in South China, Imaging Diagnosis and Interventional Center, Cancer Center, Sun Yat-sen University, Guangzhou, People's Republic of China. ${ }^{5}$ Department of Medical Statistics, First People's Hospital of Foshan Affiliated to Sun Yat-sen University, Foshan, People's Republic of China.

Received: 22 December 2014 Accepted: 30 March 2015

Published online: 18 April 2015 


\section{References}

1. Jemal A, Bray F, Center MM, Ferlay J, Ward E, Forman D. Global cancer statistics. CA Cancer J Clin. 2011;61:69-90.

2. Marks JE, Phillips JL, Menck HR. The National Cancer Data Base report on the relationship of race and national origin to the histology of nasopharyngeal carcinoma. Cancer. 1998;83:582-8.

3. Chen L, Mao YP, Xie FY, Liu LZ, Sun Y, Tian L, et al. The seventh edition of the UICC/AJCC staging system for nasopharyngeal carcinoma is prognostically useful for patients treated with intensity-modulated radiotherapy from an endemic area in China. Radiother Oncol. 2012;104:331-7.

4. Chien YC, Chen JY, Liu MY, Yang HI, Hsu MM, Chen CJ, et al. Serologic markers of Epstein-Barr virus infection and nasopharyngeal carcinoma in Taiwanese men. N Engl J Med. 2001;345:1877-82.

5. Cao SM, Liu Z, Jia WH, Huang QH, Liu Q, Guo X, et al. Fluctuations of epstein-barr virus serological antibodies and risk for nasopharyngeal carcinoma: a prospective screening study with a 20-year follow-up. PLoS One. 2011;6:e19100

6. Lee N, Xia P, Quivey JM, Sultanem K, Poon I, Akazawa C, et al. IntensityModulated radiotherapy in the treatment of nasopharyngeal carcinoma: an update of the UCSF experience. Int J Radiat Oncol Biol Phys. 2002;53:12-9.

7. Ng WT, Lee MC, Hung WM, Choi CW, Lee KC, Chan OS, et al. Clinical outcomes and patterns of failure after intensity-modulated radiotherapy for nasopharyngeal carcinoma. Int J Radiat Oncol Biol Phys. 2011;79:420-8.

8. Lin SJ, Pan JJ, Han L, Guo QJ, Hu CR, Zong JF, et al. Update report of nasopharyngeal carcinoma treated with reduced-volume intensity-modulated radiation therapy and hypothesis of the optimal margin. Radiother Oncol. 2014;110:385-9.

9. Edge SB, Compton CC. The American Joint Committee on Cancer: the $7^{\text {th }}$ edition of the AJCC cancer staging manual and the future of TNM. Ann Surg Oncol. 2010;17:1471-4.

10. Pan JJ, Xu YJ, Qiu SF, Zong JF, Guo QJ, Zhang Y, et al. A comparison between the Chinese 2008 and the 7th edition AJCC staging systems for nasopharyngeal carcinoma. Am J Clin Oncol. In press.

11. Chua DT, Sham JS, Kwong DL, Tai KS, Wu PM, Lo M, et al. Volumetric analysis of tumor extent in nasopharyngeal carcinoma and correlation with treatment outcome. Int J Radiat Oncol Biol Phys. 1997;39:711-9.

12. Chong VF, Zhou JY, Khoo JB, Chan KL, Huang J. Correlation between MR imaging-derived nasopharyngeal carcinoma tumor volume and TNM system. Int J Radiat Oncol Biol Phys. 2006;64:72-6.

13. Liang SB, Deng YM, Zhang N, Lu RL, Zhao H, Chen HY, et al. Prognostic significance of maximum primary tumor diameter in nasopharyngeal carcinoma. BMC Cancer. 2013;13:260.

14. Lai SZ, Li WF, Chen L, Luo W, Chen YY, Liu LZ, et al. How does intensitymodulated radiotherapy versus conventional two-dimensional radiotherapy influence the treatment results in nasopharyngeal carcinoma patients? Int J Radiat Oncol Biol Phys. 2011;80:661-8.

15. Peng G, Wang T, Yang KY, Zhang S, Zhang T, Li Q, et al. Prospective, randomized study comparing outcomes and toxicities of intensitymodulated radiotherapy vs. conventional two-dimensional radiotherapy for the treatment of nasopharyngeal carcinoma. Radiother Oncol. 2012;104:286-93.

16. Chan AT, Leung SF, Ngan RK, Teo PM, Lau WH, Kwan WH, et al. Overall survival after concurrent cisplatin-radiotherapy compared with radiotherapy alone in locoregionally advanced nasopharyngeal carcinoma. J Natl Cancer Inst. 2005;97:536-9.

17. Lin JC, Jan JS, Hsu CY, Liang WM, Jiang RS, Wang WY. Phase III study of concurrent chemoradiotherapy versus radiotherapy alone for advanced nasopharyngeal carcinoma: positive effect on overall and progression-free survival. J Clin Oncol. 2003;21:631-7.

18. Langendijk JA, Leemans CR, Buter J, Berkhof J, Slotman BJ. The additiona value of chemotherapy to radiotherapy in locally advanced nasopharyngeal carcinoma: a meta-analysis of the published literature. J Clin Oncol. 2004;22:4604-12.

19. Zhang N, Liang SB, Deng YM, Lu RL, Chen HY, Zhao H, et al. Primary tumor regression speed after radiotherapy and its prognostic significance in nasopharyngeal carcinoma: a retrospective study. BMC Cancer. 2014;14:136.

20. Sun XM, Su SF, Chen CY, Han F, Zhao C, Xiao WW, et al. Long-term outcomes of intensity-modulated radiotherapy for 868 patients with nasopharyngeal carcinoma: an analysis of survival and treatment toxicities. Radiother Oncol. 2014;110:398-403.
21. Zhou GQ, Yu XL, Chen M, Guo R, Lei Y, Sun Y, et al. Radiation-induced temporal lobe injury for nasopharyngeal carcinoma: a comparison of intensity-modulated radiotherapy and conventional two-dimensional radiotherapy. PLoS One. 2013;8:e67488.

22. Li WF, Sun Y, Mao YP, Chen L, Chen YY, Chen M, et al. Proposed lymph node staging system using the International Consensus Guidelines for lymph node levels is predictive for nasopharyngeal carcinoma patients from endemic areas treated with intensity modulated radiation therapy. Int J Radiat Oncol Biol Phys. 2013;86:2492-56.

23. Zong JF, Lin SJ, Chen YB, Wang BY, Xiao YP, Lin J, et al. Does MRI-detected cranial nerve involvement affect the prognosis of locally advanced nasopharyngeal carcinoma treated with intensity modulated radiotherapy? PLoS One. 2014;9:e100571.

24. Lartigau E, Le Ridant AM, Lambin P, Weeger P, Martin L, Sigal R, et al. Oxygenation of head and neck tumors. Cancer Am Cancer Soc. 1993;71:2319-25

25. Chen MK, Chen TH, Liu JP, Chang CC, Chie WC. Better prediction of prognosis for patients with nasopharyngeal carcinoma using primary tumor volume. Cancer. 2004;100:2160-6.

26. Lee CC, Huang TT, Lee MS, Hsiao SH, Lin HY, Su YC, et al. Clinical application of tumor volume in advanced nasopharyngeal carcinoma to predict outcome. Radiat Oncol. 2010;5:2-6.

27. Guo R, Sun $Y, Y u X L$, Yin WJ, Li WF, Chen YY, et al. Is primary tumor volume still a prognostic factor in intensity modulated radiation therapy for nasopharyngeal carcinoma? Radiother Oncol. 2012;104:294-9.

\section{Submit your next manuscript to BioMed Central and take full advantage of:}

- Convenient online submission

- Thorough peer review

- No space constraints or color figure charges

- Immediate publication on acceptance

- Inclusion in PubMed, CAS, Scopus and Google Scholar

- Research which is freely available for redistribution 